\title{
Viability and infectivity of Toxoplasma gondii tachyzoites exposed to Butanedione monoxime
}

\author{
Sara Bajelan $^{1} \cdot$ Mohammad Saleh Bahreini $^{1} \cdot$ Qasem Asgari $^{1}$ (D) Fattaneh Mikaeili $^{1}$
}

Received: 4 May 2020/Accepted: 5 August 2020/Published online: 17 August 2020

(C) Indian Society for Parasitology 2020

\begin{abstract}
The most important pathogenesis factor in the Apicomplexa parasites is invasion to the host cell. Given the inhibitory role of Butanedione Monoxime (BDM) on myosin-actin interaction, this study aimed to investigate the effects of this molecule on the vitality and infectivity of Toxoplasma tachyzoites in order to provide a new option for vaccine development. The tachyzoites of the RH strain of Toxoplasma gondii were exposed to different concentrations $(1,2,4,8,16,32,64$, and $128 \mu \mathrm{g} / \mathrm{mL})$ of BDM, and mortality effect was assessed by flow cytometry. Then, the penetration ability of the tachyzoites was investigated in HeLa and macrophage cell lines. The infectivity of exposed tachyzoites to BDM were also investigated in mice through following up and detecting the etiological factor. The highest percentage of mortality $(72.69 \%)$ was seen in the tachyzoites exposed to $128 \mu \mathrm{g} / \mathrm{mL}$ of the compound. The tachyzoites exposed to 32, 64, and $128 \mu \mathrm{g} /$ $\mathrm{mL}$ of BDM began the proliferation in HeLa cells after $48 \mathrm{~h}$, while this proliferation was initiated within $24 \mathrm{~h}$ in macrophage cells. All the mice inoculated with the BDMtreated tachyzoites died after 13 days. The mean survival time of the mice receiving tachyzoites exposed to $128 \mu \mathrm{g} /$ $\mathrm{mL}$ of BDM was 12.4 days, which was significantly different from the negative control group $(p=0.001)$ BDM, as the inhibitor of myosin-actin interaction, and other substances that block the entry of parasites into cells may be suitable candidates for vaccine production against
\end{abstract}

Qasem Asgari

asgarig@sums.ac.ir

1 Department of Parasitology and Mycology, School of Medicine, Shiraz University of Medical Sciences, Shiraz, Iran
Toxoplasma. Yet, future studies are required to be conducted on the issue.

Keywords Toxoplasma gondii - Infectivity · Butanedione monoxime $\cdot$ Cell culture
Abbreviations
LAV Live attenuated vaccines
BDM Butanedione monoxime
PBS Phosphate buffered saline
FCS Fetal calf serum
PMN Polymorphonuclear neutrophil

\section{Introduction}

Toxoplasmosis, a parasitic infection caused by Toxoplasma gondii, has been considered to be one of the most common parasitic infections among warm-blooded vertebrates, including humans. Based on serological findings, one-third of adult populations have been estimated to be infected with this parasite worldwide (Hill and Dubey 2018; Zhou et al. 2011). Toxoplasmosis in pregnant women precisely clarifies medical importance. In the first time of infection in pregnant women, the parasite may pass through the placenta, infect the fetus, and result in congenital abnormalities in the central nervous system and eyes and abortion in some cases (Arefkhah et al. 2019b). In addition, chronic or acute Toxoplasma infections in immunocompromised patients, without therapy, may cause life-threatening consequences (Arefkhah et al. 2019a; Carruthers and Suzuki 2007; Gatkowska et al. 2006). 
Despite the medical importance of this infection, few medicines have been shown to be effective. Pyrimethamine along with sulfadiazine as common drugs have side effects, such as teratogenic effects on the fetus, bone marrow suppression, kidney disorders, and hematological toxicity (Ben-Harari et al. 2017; Mandelbrot et al. 2018). Toxovax is the only vaccine produced for the prevention of Toxoplasma infection, which is only applicable to veterinary medicines. This vaccine acts as an inhibitor of tissue cyst formation and transmission to the fetus and, unfortunately, has not shown efficacy in humans (Li and Zhou 2018; Verma and Khanna 2013).

In Apicomplexa phylum, the most important factor in the pathogenesis of parasites is invasion to the host cell. The active entrance and movement mechanisms are based on gliding on solid materials. The external part serves as a ligand and attaches to the external surfaces. Its intracellular portion is located in the space between the plasma membrane and inner membrane complex and interacts with the actin of actomyosin motor via the tetrameric aldolase enzyme (Jewett and Sibley 2003; Meissner et al. 2013). The anterior myosin of the parasite interacts with the short actin filaments and the distal part attaches to the inner membrane complex through Myo A tail. The anterior myosin part moves on the actin filaments. The terminal part of myosin is fixed on the membrane complex and, consequently, the movement of the parasite occurs from the anterior towards the posterior surface (Meissner et al. 2013; Münter et al. 2009). Harnessing the entrance of the parasite into the host cell by some substances would be promising for therapeutic strategies.

Butanedione monoxime (BDM) is an inhibitory organic pharmaceutic molecule (Del Carmen et al. 2009; Ostap 2002), which interacts with myosin ATPase and hampers various myosin functions. However, it does not affect the actin filaments. As BDM can also block the entrance of Toxoplasma into the host cell, it reduces the number of intracellular parasites at a specific dose (Delbac et al. 2001; Wetzel et al. 2005). Penetration of Toxoplasma tachyzoites into the host cells can be inhibited by treatment of tachyzoites with different concentrations of BDM, an inhibitor of myosin-actin interaction, as a Live Attenuated Vaccines (LAV), therefore the present study was conducted to determine the mortality and infectivity effects of this compound on Toxoplasma in order to provide a new vaccine.

\section{Materials and methods}

\section{Animals}

In this study, fifty 6-8-week-inbred BALB/c mice (weight 22-25 g) were provided by Pasteur Institute, Tehran, Iran. The animals were kept at $22^{\circ} \mathrm{C}$ and $40-50 \%$ humidity and had access to standard food, water, and ventilation at the Laboratory Animal Center of Shiraz University of Medical Sciences, Shiraz, Iran. During the experiments from March 2017 to February 2018, the animals were embedded in cages and maintained under controlled conditions. The experiments were undertaken based on the guidelines for laboratory animals and Ethics Committee of Shiraz University of Medical Sciences.

\section{Parasites}

The virulent RH strain of $T$. gondii was obtained from Tehran University of Medical Sciences, Tehran, Iran. Tachyzoites of the RH strain of $T$. gondii were maintained by serial intraperitoneal passages in BALB/c inbred mice. Tachyzoites were collected $96 \mathrm{~h}$ after inoculation of $2 \times 10^{5}$ parasites in the mice by repeated flushing in the peritoneal cavity using Phosphate Buffered Saline (PBS) at $\mathrm{pH}=7.2$. Then, the tachyzoites were collected and centrifuged at $200 \mathrm{~g}$ for $5 \mathrm{~min}$ in order to remove peritoneal cells and cellular debris. The supernatant was collected and centrifuged at $800 \mathrm{~g}$ for $10 \mathrm{~min}$. The pellet, enriched with parasite tachyzoites, was rewashed with PBS and used in all experiments (Asgari et al. 2015).

\section{The direct effect of Butanedione monoxime on tachyzoites assessed by the flow cytometry method}

$2 \times 10^{5}$ of tachyzoites were exposed to different concentrations $(1,2,4,8,16,32,64$, and $128 \mu \mathrm{g} / \mathrm{mL})$ of BDM and then, the tubes were incubated at $4{ }^{\circ} \mathrm{C}$ for $3 \mathrm{~h}$. After incubation, propidium iodide (Sigma Company, USA) was added at the final concentration of $50 \mu \mathrm{g} / \mathrm{mL}$ to each tube and the staining procedure was done in dark environment at $4{ }^{\circ} \mathrm{C}$ for $30 \mathrm{~min}$.

Saponin $0.2 \%$ and intact tachyzoites were used for positive and negative controls, respectively. The mechanism of this staining is based on the acceptance of the dye by the DNA of the nucleus, which is due to cell death and apoptosis. Then, the mixtures were transferred to flowcytometric polystyrene tubes. In order to set up the flow cytometer, intact tachyzoites were used and gating was performed regarding the specific size of the parasites and their distribution range. These tubes were placed in the 
FACS Calibur flow cytometer to assess the effects of different concentrations of the molecules on the vitality of the tachyzoites. The device was set to the PI color and 10-30 thousand cells were counted from each tube.

\section{Cell culture}

HeLa cells and J774 macrophage cell line were provided from the Department of Immunology, Shiraz University of Medical Sciences. A monolayer of the cells was cultivated in $5 \mathrm{~mL}$ of the RPMI 1640 medium (Sigma Company, USA) enriched with $10 \%$ heat-inactivated Fetal Calf Serum (FCS; Gibco Company, USA) and $100 \mathrm{IU} / \mathrm{mL}$ penicillin$100 \mu \mathrm{g} / \mathrm{mL}$ streptomycin (Roche Company) using $25 \mathrm{~cm}^{2}$ flasks (Corning Costar UK, UK) and was incubated in 5\% $\mathrm{CO}_{2}$ at $37{ }^{\circ} \mathrm{C}$ and $>80 \%$ humidity. The cells were routinely subcultured every 3 days by trypsinization and washing with PBS $(\mathrm{pH}=7.2)$.

\section{Cell culture of Toxoplasma gondii tachyzoites exposed to Butanedione monoxime}

To determine the effects of different dosages of the molecule on the ability of the tachyzoites to attack the cells, the parasite was cultivated in a HeLa cell and J774 cell line culture. After $70 \%$ confluency of the cell line in a monolayer condition, the tachyzoites exposed to different concentrations were added to the cell culture at 3:1 ratio. The flasks were then incubated at $37{ }^{\circ} \mathrm{C},>80 \%$ humidity, and $5 \% \mathrm{CO}_{2}$. The cultures were checked by an invert microscope with 24-h intervals and the results of growth and propagation of the parasite were recorded and compared to the control sample. The load of the parasite was calculated using the following formula:

Load of parasite $=$ the mean number of tachyzoites in a host cell determined in 100 infected cells $\times$ the ratio of the infected cells to 100 cells in the cell culture.

\section{The exposed tachyzoites infectivity in animals}

A total of 50 mice were divided into 10 groups. Then, $2 \times 10^{5}$ tachyzoites treated with different concentrations $(1,2,4,8,16,32,64$, and $128 \mu \mathrm{g} / \mathrm{mL})$ of the compound were subcutaneously injected into the mice. Also, five mice as the positive control group were infected by intact tachyzoites and then treated with sulfadiazine and for the negative control group, intact tachyzoites were injected into five mice without any treatment. The mice were followed up for one month. Once the mice died, the liver touch smears were prepared and stained by Giemsa staining to detect the etiological factor.

\section{Data analysis}

The data were entered into the SPSS software (version 16, Chicago, USA) and were analyzed using Mann-Whitney non-parametric test. $P<0.05$ was considered to be statistically significant.

\section{Results}

The flow cytometry results of Toxoplasma tachyzoites exposed to saponin, as positive control, and different doses of BDM have been shown in Figs. 1 and 2. Accordingly, the highest rate of mortality $(72.69 \%)$ was seen at the $128 \mu \mathrm{g} / \mathrm{mL}$ concentration of BDM.

The tachyzoites exposed to different concentrations of BDM were cultured in the HeLa cell line and their infectivity and invasion ability were examined using invert microscopy. As fully illustrated in Fig. 3 and Table 1, the tachyzoites proliferation was observed after $48 \mathrm{~h}$ in the exposed parasites to 32,64 , and $128 \mu \mathrm{g} / \mathrm{mL}$ of BDM. However, the proliferation of the exposed tachyzoites to different concentrations of the molecule was seen after $24 \mathrm{~h}$ in the macrophage cells. The load of the tachyzoites exposed to different dosages of BDM in the HeLa cell culture for $48 \mathrm{~h}$ has been presented in Fig. 3.

The results indicated that all groups of mice died after 13 days. Hence, the infectivity in the tachyzoites of Toxoplasma gondii exposed to different dosages of this compound was maintained.

Regarding the results of the Log-Rank test, the mice receiving the tachyzoites exposed to $128 \mu \mathrm{g} / \mathrm{mL}$ of $\mathrm{BDM}$ had significant longevity (12.4 days) compared to the control group $(p<0.001)$ (Fig. 4).

\section{Discussion}

This study aimed to evaluate the effect of BDM on the entrance of Toxoplasma gondii tachyzoites. In this protocol, tachyzoites were exposed to different concentrations of BDM and then, the invasive power of these tachyzoites was assessed. In the flow cytometry analysis of different concentrations of the molecule on Toxoplasma gondii tachyzoites using PI staining, the highest mortality rate was observed in $128 \mu \mathrm{g} / \mathrm{mL}$. In vivo results showed a significant difference between the case and negative control groups regarding the 12.4-day mean survival rate at the $128 \mu \mathrm{g} / \mathrm{mL}$ concentration of the molecule. The in vitro and in vivo results were correlated. Moreover, the flow cytometry results showed that the mortality of tachyzoites exposed to $128 \mu \mathrm{m} / \mathrm{mL}$ of BDM was $72 \%$. Thus, the high 

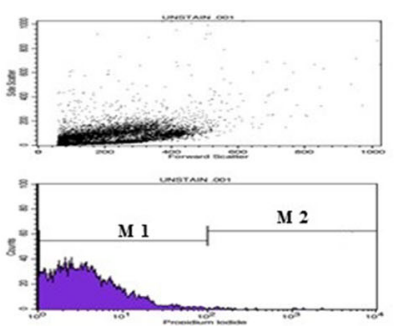

Unstained
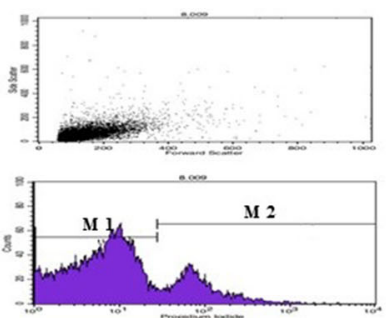

$8 \mu \mathrm{g} / \mathrm{ml}$
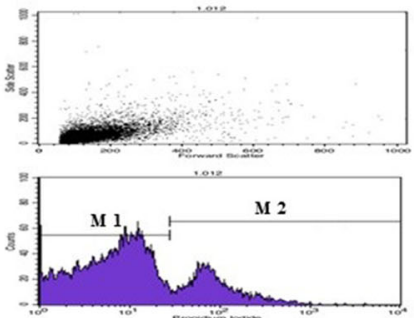

$1 \mu \mathrm{g} / \mathrm{ml}$
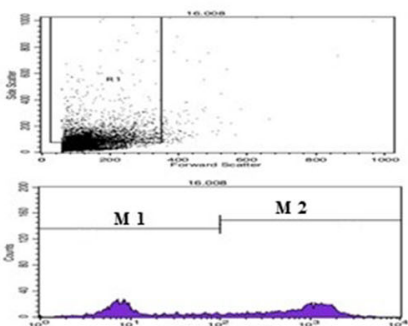

$16 \mu \mathrm{g} / \mathrm{ml}$
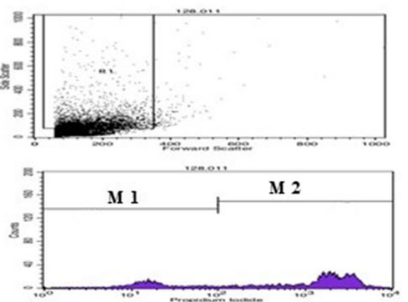

$128 \mu \mathrm{g} / \mathrm{ml}$
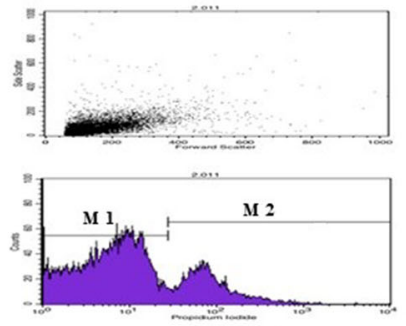

$2 \mu \mathrm{g} / \mathrm{ml}$
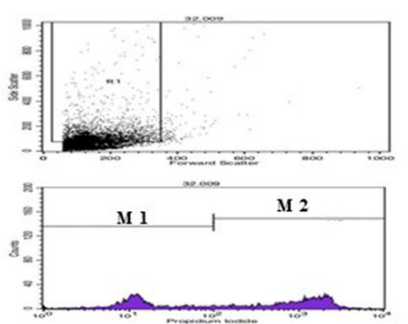

$32 \mu \mathrm{g} / \mathrm{ml}$

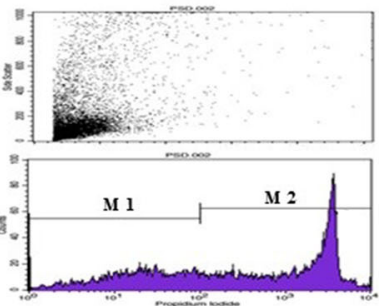

Saponin
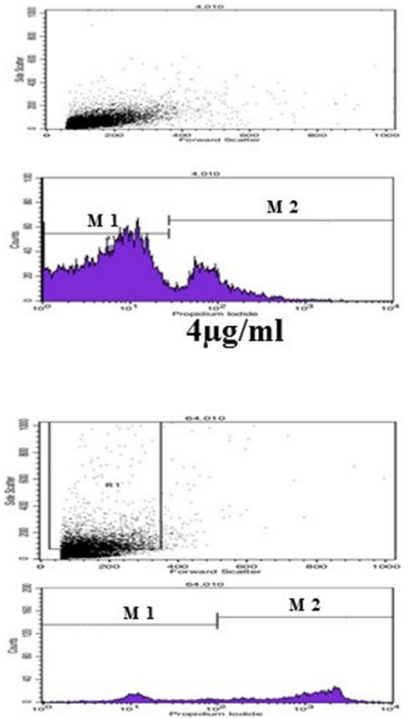

$64 \mu \mathrm{g} / \mathrm{ml}$

Fig. 1 The flow cytometry results of Toxoplasma tachyzoites exposed to different doses of Butanedione monoxime

Fig. 2 Relationship between different doses of Butanedione monoxime and percentage of mortality of Toxoplasma tachyzoites with flow cytometry

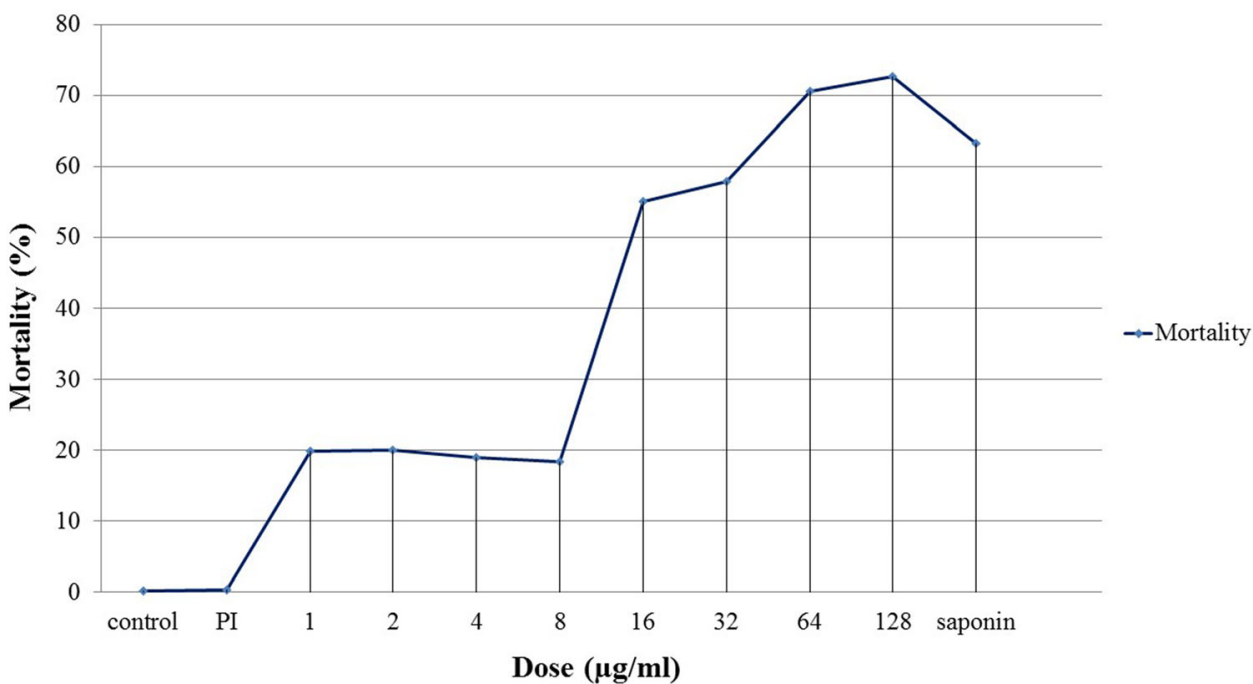

longevity of the mice belonged to the lower vitality of the tachyzoites.

The most important factor in Apicomplexan parasites is their ability to penetrate into host cells (Bargieri et al. 2014). BDM is an inhibitory organic pharmaceutical molecule (Del Carmen et al. 2009; Ostap 2002), which interacts with myosin ATPase and hampers various functions of myosin. However, it does not affect the actin filaments. In addition to an inhibitory role, these therapeutic agents inhibit the interaction between myoA and actin. It 
Fig. 3 The load of tachyzoites cells exposed to different doses of Butanedione monoxime in HeLa cell culture after $48 \mathrm{~h}$

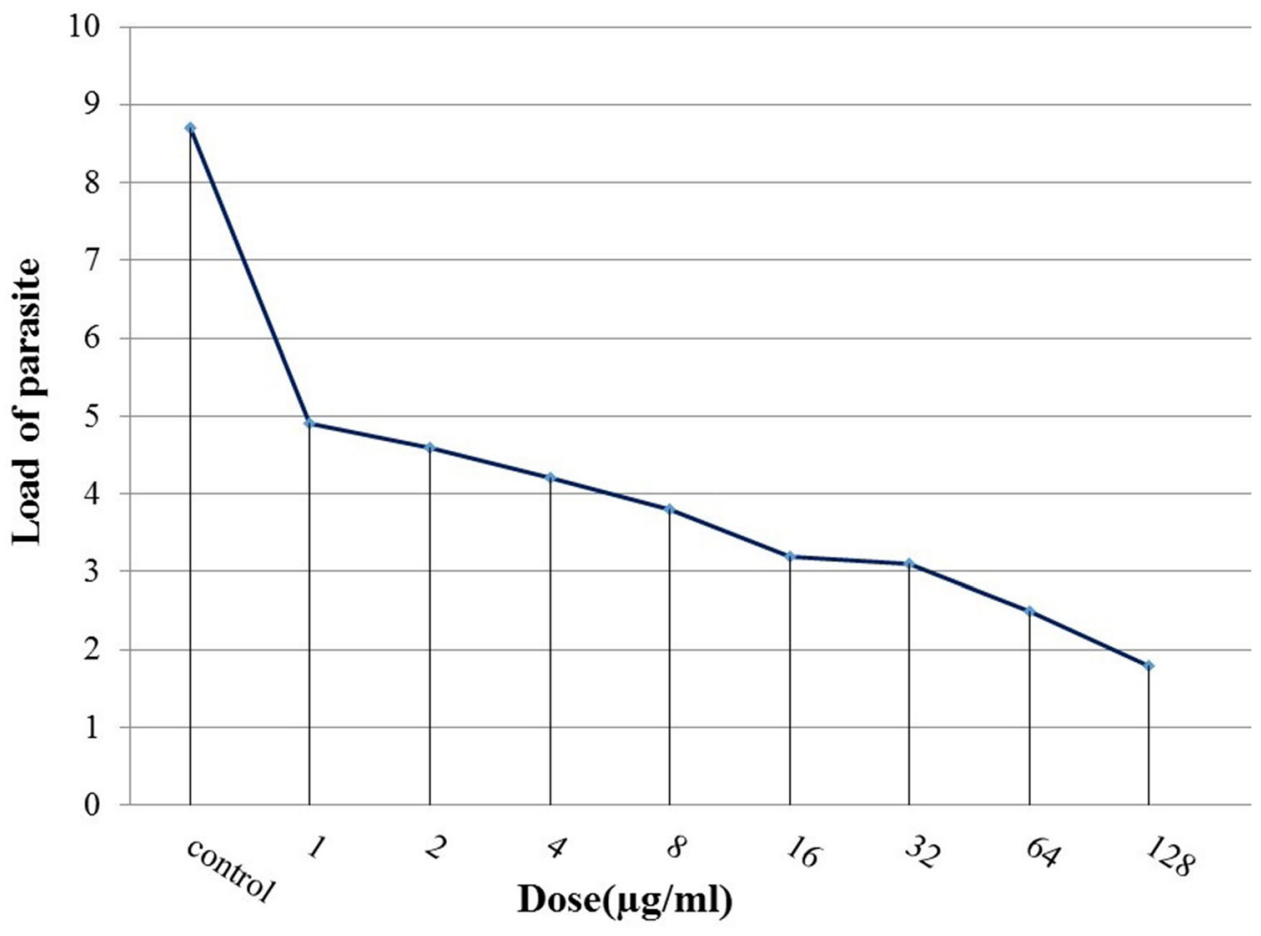

Table 1 The results of the proliferation of tachyzoites exposed to Butanedione monoxime in HeLa and macrophage cell line culture at different times; positive control: intact tachyzoites, negative control: not infected cells

Cell lines

\begin{tabular}{|c|c|c|c|c|c|}
\hline \multicolumn{3}{|l|}{ HeLa } & \multicolumn{3}{|l|}{ Macrophage (J774) } \\
\hline Tachyzoites exposed to BDM & Positive control & Negative control & Tachyzoites exposed to BDM & Positive control & Negative contro \\
\hline \multicolumn{6}{|l|}{ ration time after infection } \\
\hline- & + & - & - & + & - \\
\hline- & + & - & + & + & - \\
\hline+ & + & - & + & + & - \\
\hline+ & + & - & + & + & - \\
\hline
\end{tabular}

seems, BMD blocks the entrance of Toxoplasma into the host cell due to above inhibitory path (Delbac et al. 2001; Wetzel et al. 2005). Furthermore, it has been shown that, BDM as a myosin ATPase inhibitor impedes the invasion of Plasmodium species (Campino et al. 2018; Siden-Kiamos et al. 2006). However, a lower concentration of BDM can inhibit the invasion of Plasmodium species (Morales et al. 2018).

In this study, parasite proliferation was observed at 32, 64, and $128 \mu \mathrm{g} / \mathrm{mL}$ concentrations of BDM after $48 \mathrm{~h}$ in the HeLa cell culture, while the entrance of the parasite into the macrophages did not need time. The reason for the delayed invasion to HeLa cells compared to macrophages was related to their different cell entrance mechanisms. The entrance of the parasite into HeLa cells occurs in an active form, but macrophages have a phagocytosis system for cell entrance.

A couple of studies have been conducted recently to evaluate the effects of BDM on a range of parasitic infections. In the research carried out by Dabrowolski et al., BDM was suggested as an inhibitor of Toxoplasma gondii motility and was introduced as an entrance/motilitylimiting agent for Toxoplasma gondii. This agent counteracted the microneme secretions and, consequently, impeded the entrance of the parasite, which was consistent with the current study findings (Dobrowolski et al. 1997). Ostap et al. measured the activities of myosin 2 ATPase in skeletal muscle, human myosin, Acanthamoeba myosin, and avian isoform of myosin at $0-40 \mathrm{Mm}$ concentrations. BDM represented inhibitory effects merely on the skeletal muscle myosin-2 (Ostap 2002). 
Fig. 4 The mean survival time of mice receiving tachyzoites exposed to the different doses of Butanedione monoxime

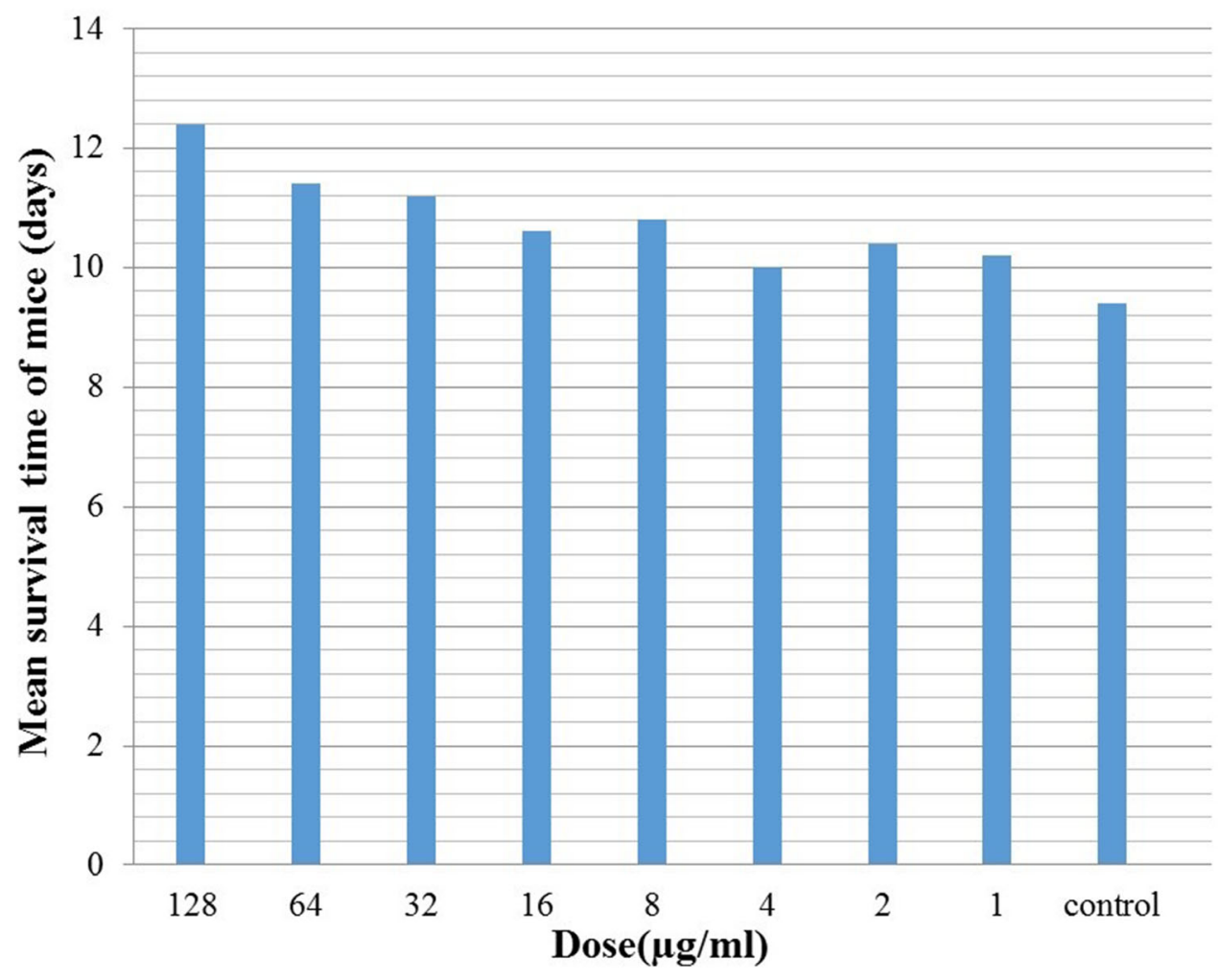

Streinberg et al. evaluated the effects of BDM on the morphological characteristics of Saccharomyces species and indicated a gradual reduction in the formation and growth of colonies on Agar medium at 1-30 $\mu \mathrm{m}$ BDM concentrations (Steinberg and McIntosh 1998). In another study by Esnault et al., BDM decreased inflation and budding capability of Aspergillus Fumigatus species and led to impaired cell wall disruption (Esnault et al. 1999). Furthermore, Schmidt and Wetzel et al. showed that the motility and invasion of Cryptosporidium Parvum were hindered by Cytochalasin D and BDM, respectively (Wetzel et al. 2005). In the study by Kiamus and Pinder also, BDM was used as the inhibitor of myosin dysfunction and decreased the motility of Plasmodium berghei ookinetes (Siden-Kiamos et al. 2006). Moreover, Kivisto et al. reported that 2,3-BDM, as a myosin II ATPase, could inhibit cardiac contraction in murine ventricular cardiomyocyte media culture (Kirschner Peretz et al. 2017; Kivistö et al. 1995). However, the results of several studies showed that BDM had additional effects, such as altering L-type $\mathrm{Ca}^{2+}$ channel activity, SR Ca ${ }^{2+}$ load (Gwathmey et al. 1991), cardiac action potential characteristics and conduction (Kettlewell et al. 2004; Lou et al. 2011), and induction of Polymorphonuclear Neutrophils (PMNs) polarity by chemotactic agents (Seveau et al. 1997).

By comparing the results of these studies with the studies mentioned above, it could be concluded that using the parasite paralysis method is a suitable candidate for a toxoplasma vaccine and create a protective response. Also, BDM molecule could be useful in advancing this method due to its effect on inhibiting the toxoplasma's motor system.

The limitations of this study are that protective response generated by the tachyzoites exposed to different concentrations of BDM was only evaluated by examining the Survival time of the mice, which can be done more accurately by examining immune factors such as cytokines and interleukins in future research.

\section{Conclusions}

The tachyzoites exposed to high doses of BDM showed a low infectivity compared to the control group. Nonetheless, obtaining a more appropriate dose as well as a more effective material to paralyze the parasite as a vaccine target could be a good subject for future studies in order to introduce an effective vaccine.

Acknowledgements The authors would like to thank Ms. A. Keivanshekouh at the Research Improvement Center of Shiraz University of Medical Sciences for improving the use of English in the manuscript.

Authors' contributions QA and FM coordinated in designing the project and providing financial supports. SB and MSB Performed the experiments and analyzed the data. QA, SB and MSB carried out 
drafted the original manuscript and all authors read and approved the final version of the manuscript.

Funding This study was financially supported by the Vice-chancellor for Research Affairs of Shiraz University of Medical Sciences, Shiraz, Iran (Grant No. 95-01-01-12970).

Availability of data and materials The dataset used and/or analyzed during the current study is available from the corresponding author upon reasonable request.

\section{Compliance with ethical standards}

Competing interests The authors declare that they have no competing interests.

Ethics approval and consent to participate This study was approved by the Ethics Committee of Shiraz University of Medical Sciences (IR.SUMS.REC.1396.S653). The guideline of the Institutional Animal Care and Ethics Committee of Animal Experimentation, Shiraz University of Medical Sciences was used to work on animal models in this study.

Consent for publication Not applicable.

\section{References}

Arefkhah N et al (2019a) Seroprevalence and risk factors of Toxoplasma gondii infection among cancer and hemodialysis patients in southwest Iran. Clin Epidemiol Glob Health 7(4):596-599

Arefkhah N, Pourabbas B, Asgari Q, Moshfe A, Mikaeili F, Nikbakht G, Sarkari B (2019b) Molecular genotyping and serological evaluation of Toxoplasma gondii in mothers and their spontaneous aborted fetuses in Southwest of Iran. Comp Immunol Microbiol Infect Dis 66:101342

Asgari Q, Keshavarz H, Rezaeian M, Sadeghpour H, Miri R, Motazedian MH (2015) Anti-toxoplasma activity of 2-(naphthalene-2- $\gamma$ lthiol)-1H indole. Iran J Parasitol 10(2):171

Bargieri D, Lagal V, Andenmatten N, Tardieux I, Meissner M, Menard R (2014) Host cell invasion by apicomplexan parasites: the junction conundrum. PLoS Pathog 10(9):e1004273

Ben-Harari RR, Goodwin E, Casoy J (2017) Adverse event profile of pyrimethamine-based therapy in toxoplasmosis: a systematic review. Drugs R\&D 17(4):523-544

Campino $S$ et al (2018) A forward genetic screen reveals a primary role for Plasmodium falciparum reticulocyte binding protein Homologue $2 \mathrm{a}$ and $2 \mathrm{~b}$ in determining alternative erythrocyte invasion pathways. PLoS Pathog 14(11):e1007436

Carruthers VB, Suzuki Y (2007) Effects of Toxoplasma gondii infection on the brain. Schizophr Bull 33(3):745-751

Del Carmen MG, Mondragon M, Gonzalez S, Mondragon R (2009) Induction and regulation of conoid extrusion in Toxoplasma gondii. Cell Microbiol 11(6):967-982

Delbac F et al (2001) Toxoplasma gondii myosins B/C: one gene, two tails, two localizations, and a role in parasite division. J Cell Biol 155(4):613-624

Dobrowolski JM, Carruthers VB, Sibley LD (1997) Participation of myosin in gliding motility and host cell invasion by Toxoplasma gondii. Mol Microbiol 26(1):163-173

Esnault K, el Moudni B, Bouchara J-P, Chabasse D, Tronchin G (1999) Association of a myosin immunoanalogue with cell envelopes of Aspergillus fumigatus Conidia and its participation in swelling and germination. Infect Immun 67(3):1238-1244
Gatkowska J, Hiszczynska-Sawicka E, Kur J, Holec L, Dlugonska H (2006) Toxoplasma gondii: an evaluation of diagnostic value of recombinant antigens in a murine model. Exp Parasitol 114(3):220-227

Gwathmey J, Hajjar R, Solaro R (1991) Contractile deactivation and uncoupling of crossbridges: effects of 2, 3-butanedione monoxime on mammalian myocardium. Circ Res 69(5):1280-1292

Hill DE, Dubey JP (2018) Toxoplasma gondii. In: Foodborne Parasites. Springer, pp 119-138

Jewett TJ, Sibley LD (2003) Aldolase forms a bridge between cell surface adhesins and the actin cytoskeleton in apicomplexan parasites. Mol Cell 11(4):885-894

Kettlewell S, Walker N, Cobbe S, Burton F, Smith G (2004) The electrophysiological and mechanical effects of 2,3-butane-dione monoxime and cytochalasin-D in the Langendorff perfused rabbit heart. Exp Parasitol 89(2):163-172

Kirschner Peretz N et al (2017) A method sustaining the bioelectric, biophysical, and bioenergetic function of cultured rabbit atrial cells. Front Physiol 8:584

Kivistö T, Mäkiranta M, Oikarinen E-L, Karhu S, Weckström M, Sellin L (1995) 2, 3-Butanedione monoxime (BDM) increases initial yields and improves long-term survival of isolated cardiac myocytes. Jpn J Physiol 45(1):203-210

Li Y, Zhou H (2018) Moving towards improved vaccines for Toxoplasma gondii. Expert Opin Biol Ther 18(3):273-280

Lou Q, Li W, Efimov IR (2011) The role of dynamic instability and wavelength in arrhythmia maintenance as revealed by panoramic imaging with blebbistatin vs. 2, 3-butanedione monoxime. Am J Physiol-Heart C 302(1):H262-H269

Mandelbrot L et al (2018) 31: Congenital toxoplasmosis prevention by pyrimethamine-sulfadiazine vs. spiramycin, a randomized trial. Am J Obstet Gynecol 218(1):S25

Meissner M, Ferguson DJ, Frischknecht F (2013) Invasion factors of apicomplexan parasites: essential or redundant? Curr Opin Microbiol 16(4):438-444

Morales L, Hernández P, Chaparro-Olaya J (2018) Systematic comparison of strategies to achieve soluble expression of Plasmodium falciparum recombinant proteins in E. coli. Mol Biotechnol 60(12):887-900

Münter S et al (2009) Plasmodium sporozoite motility is modulated by the turnover of discrete adhesion sites. Cell Host Microbe 6(6):551-562

Ostap EM (2002) 2, 3-Butanedione monoxime (BDM) as a myosin inhibitor. J Muscle Res Cell Motil 23(4):305-308

Seveau S, Lopez S, Lesavre P, Guichard J, Cramer EM, HalbwachsMecarelli L (1997) Leukosialin (CD43, sialophorin) redistribution in uropods of polarized neutrophils is induced by CD43 cross-linking by antibodies, by colchicine or by chemotactic peptides. J Cell Sci 110(13):1465-1475

Siden-Kiamos I, Pinder JC, Louis C (2006) Involvement of actin and myosins in Plasmodium berghei ookinete motility. Mol Biochem Parasit 150(2):308-317

Steinberg G, McIntosh JR (1998) Effects of the myosin inhibitor 2, 3-butanedione monoxime on the physiology of fission yeast. Eur J Cell Biol 77(4):284-293

Verma R, Khanna P (2013) Development of Toxoplasma gondii vaccine: a global challenge. Hum Vaccin 9(2):291-293

Wetzel DM, Schmidt J, Kuhlenschmidt MS, Dubey J, Sibley LD (2005) Gliding motility leads to active cellular invasion by Cryptosporidium parvum sporozoites. Infect Immun 73(9):5379-5387

Zhou P, Chen Z, Li H-L, Zheng H, He S, Lin R-Q, Zhu X-Q (2011) Toxoplasma gondii infection in humans in China. Parasite Vector 4(1): 165

Publisher's Note Springer Nature remains neutral with regard to jurisdictional claims in published maps and institutional affiliations. 